\title{
Experimental Demonstration of an Electrostatic Orbital Angular Momentum Sorter for Electron Beams
}

\author{
Amir H. Tavabi®, ${ }^{1}$ Paolo Rosi $\odot,{ }^{2}$ Enzo Rotunno, ${ }^{3, *}$ Alberto Roncaglia $\odot,{ }^{4}$ Luca Belsito, ${ }^{4}$ \\ Stefano Frabboni, ${ }^{2,3}$ Giulio Pozzi, ${ }^{1,5}$ Gian Carlo Gazzadi, ${ }^{3}$ Peng-Han Lu, ${ }^{1,6}$ Robert Nijland, ${ }^{7}$ \\ Moumita Ghosh, ${ }^{7}$ Peter Tiemeijer $\odot,{ }^{7}$ Ebrahim Karimi $\odot,{ }^{8}$ Rafal E. Dunin-Borkowski $\odot,{ }^{1}$ and Vincenzo Grillo ${ }^{3, \dagger}$ \\ ${ }^{1}$ Ernst Ruska-Centre for Microscopy and Spectroscopy with Electrons and Peter Grünberg Institute, \\ Forschungszentrum Jülich, 52425 Jülich, Germany \\ ${ }^{2}$ Dipartimento FIM, Universitá di Modena e Reggio Emilia, 41125 Modena, Italy \\ ${ }^{3}$ Centro S3, Istituto di Nanoscienze-CNR, 41125 Modena, Italy \\ ${ }^{4}$ Istituto per la Microelettronica e i Microsistemi-CNR, 40129 Bologna, Italy \\ ${ }^{5}$ Department of Physics and Astronomy, University of Bologna, 40127 Bologna, Italy \\ ${ }^{6}$ RWTH Aachen University, 52074 Aachen, Germany \\ ${ }^{7}$ Thermo Fisher Scientific, PO Box 80066, 5600 KA Eindhoven, Netherlands \\ ${ }^{8}$ Department of Physics, University of Ottawa, Ottawa, Ontario K1N 6N5, Canada
}

(Received 29 September 2020; revised 6 December 2020; accepted 12 January 2021; published 5 March 2021)

\begin{abstract}
The component of orbital angular momentum (OAM) in the propagation direction is one of the fundamental quantities of an electron wave function that describes its rotational symmetry and spatial chirality. Here, we demonstrate experimentally an electrostatic sorter that can be used to analyze the OAM states of electron beams in a transmission electron microscope. The device achieves postselection or sorting of OAM states after electron-material interactions, thereby allowing the study of new material properties such as the magnetic states of atoms. The required electron-optical configuration is achieved by using microelectromechanical systems technology and focused ion beam milling to control the electron phase electrostatically with a lateral resolution of $50 \mathrm{~nm}$. An OAM resolution of $1.5 \hbar$ is realized in tests on controlled electron vortex beams, with the perspective of reaching an optimal OAM resolution of $1 \hbar$ in the near future.
\end{abstract}

DOI: 10.1103/PhysRevLett.126.094802

Although electron microscopy was pioneered in the early 1930s [1], the discipline continues to be developed technically and methodologically [2,3]. A modern transmission electron microscope can be used to characterize materials with sub-0.1-nm spatial resolution [4-6], to provide threedimensional microstructural and compositional information [7], to record electron energy-loss spectra with a sensitivity of a few $\mathrm{meV}[8,9]$, and to achieve new horizons in protein characterization [10,11]. One of the most significant advances has been the theoretical and experimental development of spherical aberration correction [12-14], which requires the precise manufacture, alignment, and control of the magnetic fields of electron-optical elements using computer-aided procedures and electronics. Recently, other concepts have been taken from light optics and used to explore new directions in electron-optical instrumentation.

Published by the American Physical Society under the terms of the Creative Commons Attribution 4.0 International license. Further distribution of this work must maintain attribution to the author(s) and the published article's title, journal citation, and DOI.
For example, synthetic holograms have been used to realize electron vortex beams [15-24], nondiffractive beams $[25,26]$, and new measurement schemes [27-29]. These developments have been used to introduce the component of orbital angular momentum (OAM) in the propagation direction (henceforth defined $z$ ) as a "new" degree of freedom in electron microscopy. However, the use of synthetic holograms is detrimental to electron beam coherence and intensity, motivating the use of electrostatic and magnetostatic fields to achieve electron beam control [30-32].

Here, we realize an electrostatic "sorter" for electrons that can be used to measure the OAM components of an electron beam. The $z$ component of the OAM operator is proportional to the gradient of the electron wave function with respect to the azimuthal coordinate $\theta$, according to the expression $L_{z}=-i \hbar \partial_{\theta}$. Its eigenstates are electron vortex beams, which are characterized by azimuthal phase terms and can be described in the form $e^{-i \ell \theta}$. The measurement of an OAM state is therefore equivalent to finding the value of $\ell$. We realized such a device based on two holographic phase elements fabricated from $\mathrm{SiN}$ [27]. In the present study, we demonstrate a complete setup that is based on the 
use of controllable electrostatic fields and can be retrofitted to an existing electron microscope.

An OAM sorter is intended to study an electron beam after its interaction with a sample [33-35]. Previous work focused on postselection of linear momentum after elastic and, more interestingly, inelastic scattering of electron beams [36,37]. However, little has been achieved in terms of control of the OAM final state. Electron energy-loss spectroscopy with OAM postselection could be used to assess magnetic dichroism [33-35], to discriminate multipolar transitions [38], to study two-dimensional materials [39], and for more exotic applications [40]. However, efficient and accurate measurement is required for this to become a valuable tool in materials science and cryoelectron microscopy [41].

Previous methods of OAM measurement [42-49] were based on diffraction by components such as amplitude apertures. In contrast, an OAM sorter provides decomposition of all OAM channels at the same time by making use of a conformal mapping from Cartesian to log-polar coordinates. Such a coordinate transformation permits radial and azimuthal degrees of freedom to be decoupled. Since it corresponds to a unitary change of basis, all of the components of the electron beam that have a chosen OAM value are propagated into a single region of space with virtually no loss and with superior efficiency to the use of a pitchfork hologram [50], despite the simplicity of the latter concept. Although the Stern-Gerlach-like approach described in Ref. [47] also offers a potentially near-unitary transformation, it does not decouple radial and azimuthal degrees of freedom.

Here, we describe the technical steps that are required to realize an electrostatic OAM sorter. We present preliminary results that demonstrate its successful operation, including the recording of OAM spectra in a transmission electron microscope.

Whereas an OAM sorter that is based on the use of synthetic holograms follows an equivalent light-optical holographic approach [51], McMorran et al. showed theoretically [30] that a set of electrodes is able to reproduce the required phase elements. This proposal was improved by Pozzi et al. [31], who proposed the use of additional electrodes outside the area of the beam. In addition, it was realized that all conformal mappings can be reproduced by the use of near-harmonic phase elements, such as those produced by electrodes [52]. Conformal mappings in optics involve transformations of coordinates by imparting appropriate phase gradients (and therefore inplane velocities) to wave functions and allowing them to evolve to corresponding diffraction planes. They can be achieved by using two phase elements and making use of quantum mechanical changes of basis to measure quantities such as OAM. According to theory [30,31], a first sorter element $S 1$ (or "unwrapper") can take the form of a long needle located in front of an electrostatic mirror. To a first approximation, such a needle can be modeled as a straight line of constant charge density or, equivalently, as an ellipsoid-shaped electrode that corresponds to a desired equipotential contour. An electrostatic mirror (i.e., a grounded flat electrode) should be positioned in front of the needle to ensure charge neutrality. The needle introduces a phase cuspid, which unwraps the electron beam so that an azimuthal phase gradient is transformed into a linear gradient in the stationary phase approximation. This transformation is completed in the far-field diffraction plane, where a second phase element $S 2$ (or "sorter corrector") removes the transformational phase and the beam can continue without rewrapping again. The second element can take the form of a series of in-plane parallel needleshaped electrodes of alternating opposite polarity.

It is convenient to use complex notation to describe mappings from complex coordinates $u=x+i y$ to $u^{\prime}=x^{\prime}+i y^{\prime}$, where $x, y$ and $x^{\prime}, y^{\prime}$ are orthogonal to the main propagation direction. The first conformal mapping can be expressed $u^{\prime}=s f \ln (u / L)$, where $s$ is a transformation scale parameter (approximately equivalent to the average tilt imparted by element $S 1), f$ is the focal length of the lens system between the elements, and $L$ is a characteristic length (corresponding to the length of the needle in the electrostatic case). Element $S 1$ applies a phase distribution of the form

$$
\varphi_{1}=\frac{s}{\lambda} \operatorname{Re}\left\{\mathrm{u} \ln \left(\frac{u}{L}\right)-u\right\},
$$

while element $S 2$ applies a compensating phase distribution of the form

$$
\varphi_{2}=\frac{L s}{\lambda} \operatorname{Re}\left\{\exp \left(\frac{u}{s f}\right)\right\},
$$

where Re is the real value of a function and $\lambda$ is the electron wavelength. (See the Supplemental Material [53]).

Based on this initial design, we have developed devices that can be fitted into an electron microscope. Without altering the microscope significantly, i.e., without adding lenses, we realized OAM sorter elements $S 1$ and $S 2$ using apertures that were fabricated using microelectromechanical systems (MEMS) technology, one of which was placed in the back focal plane of the objective lens and the second in a selected area diffraction (SAD) plane. The aperture holders were custom-made and equipped with housings for $4 \times 11 \mathrm{~mm}$ MEMS chips with sockets based on those used in a Thermo Fisher NanoEx-i/v specimen holder. MEMS fabrication involved two-dimensional patterning of the device electrodes using optical lithography and deep reactive ion etching of silicon-on-insulator wafers using a recessed etching isolation technique. Up to 8 electrical pins could be used inside the microscope by connecting the internal to the external sockets through the body of each aperture holder. The use of MEMS technology in a planar geometry in a restricted space required several changes from 
the theoretical geometry. Needle $S 1$ was replaced by three needles, as recently reported calculations [31] showed that astigmatism introduced by a finite needle can be compensated by the use of lateral electrodes oriented perpendicular to it. The shapes of the needles were initially defined using MEMS fabrication and then adjusted to achieve more precise (on a $50 \mathrm{~nm}$ scale) ellipsoidal shapes using focused ion beam milling. A virtually infinite series of electrodes in device $S 2$ was approximated by 11 electrodes. Smart use of a planar topology with links behind the connection pads permitted the even pins to be connected to each other, while the odd pins could be driven independently. Figure 1 shows off-axis electron holography measurements of electrically biased
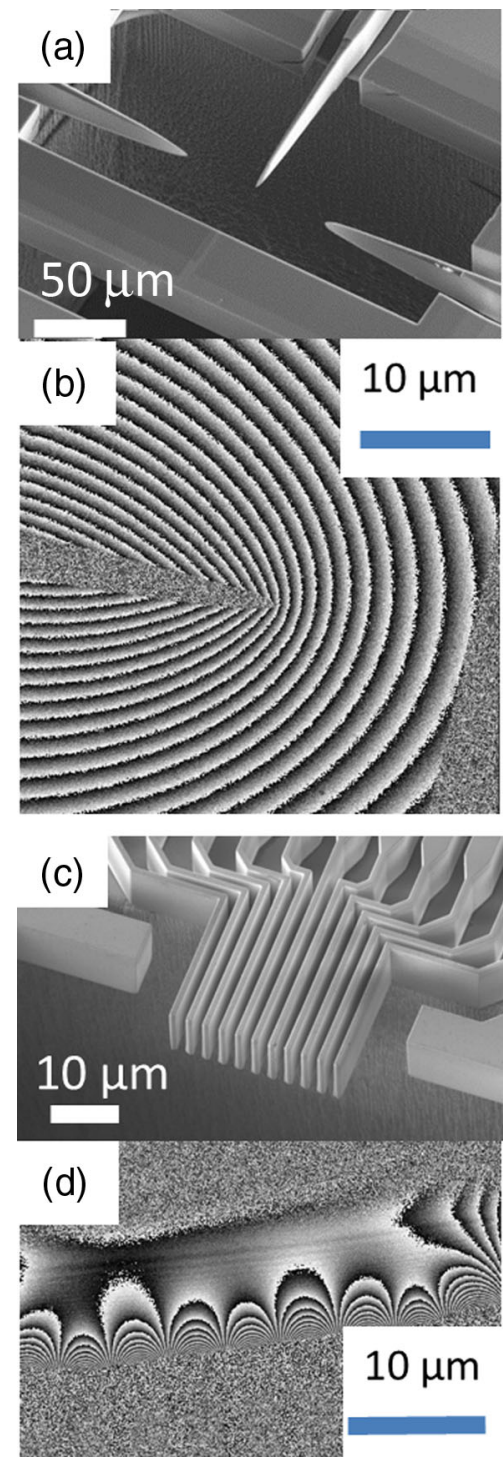

FIG. 1. (a),(c) Scanning electron microscopy images of electrostatic sorting elements $S 1$ (unwrapper) and $S 2$ (corrector). (b),(d) Electron optical phase contour maps showing projected electrostatic potentials recorded using off-axis electron holography for elements $S 1$ and $S 2$, respectively. For element $S 1$, the bias applied to the main tip was reduced to $1 \mathrm{~V}$ for this measurement.
MEMS chips inserted in the specimen plane of a standard Thermo Fisher NanoEx-i/v holder, confirming the expected phase distributions. The phase profile for $S 1$ is almost ideal. The $S 2$ phase contours are also almost ideal, in particular close to the central pins. Although the influence of truncation resulting from the use of only 11 needles is visible, it does not affect the region of the central 5 electrodes significantly.

Figure 2(a) shows a schematic diagram of the positions of the phase elements and their effect on the electron beam. Experiments were performed at $300 \mathrm{kV}$ in a Thermo Fisher Titan G2 60-300 transmission electron microscope, which is equipped with an X-FEG electron emitter and an image spherical aberration corrector. The illumination system was set to spot size 9 and the three condenser lens system of the microscope was used to achieve a probe convergence semiangle of $2 \mathrm{mrad}$ in the specimen plane. Although a specimen can be located in a standard position in the objective lens, for test purposes a synthetic hologram, which is referred to here as a "generation" hologram, was placed in the second condenser aperture plane, in order to generate a known electron beam (typically a superposition of vortex beams). The microscope was operated in "microprobe" mode, with the objective lens excited to a standard preset value. The lenses and multipoles in the image aberration corrector were switched off, in order to achieve a larger focal distance between the sorting

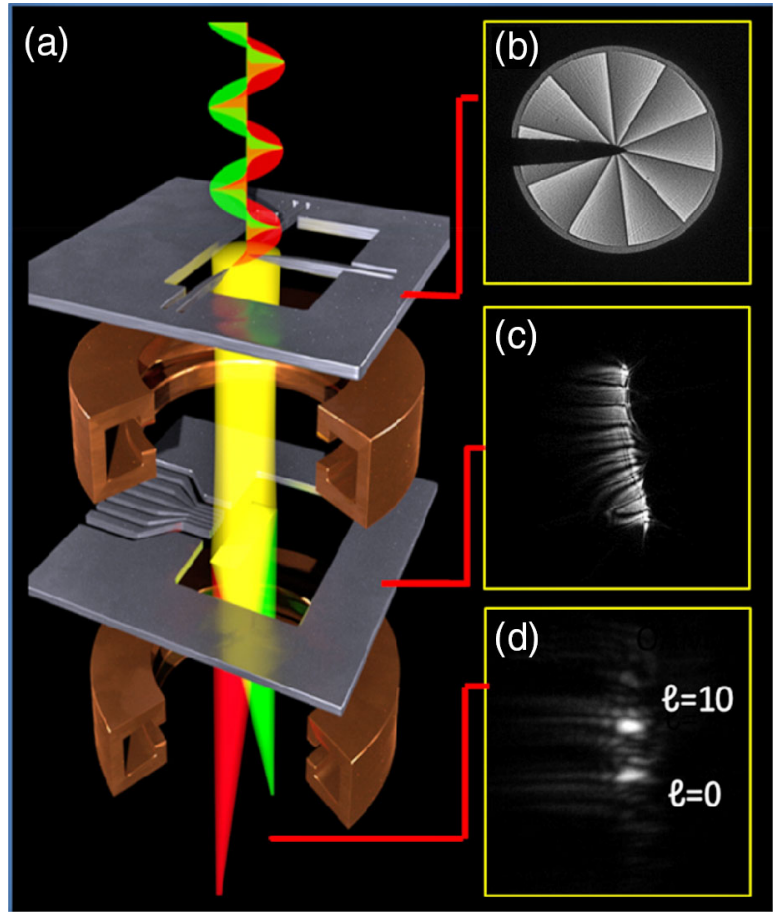

FIG. 2. (a) Schematic diagram of part of an electron microscope column that contains a sorter. (b)-(d) Experimental images showing the evolution of the electron wave function for a nominal superposition of $|\ell=10\rangle$ and $|\ell=0\rangle$, including (b) generation, (c) conformal transformation to polar coordinates, and (d) transformation into an OAM spectrum. 
elements. In this configuration, the second sorter element $S 2$ was located in the diffraction plane of the first sorter element $S 1$ (mounted in the objective aperture plane), which is conjugate to the specimen plane. An OAM spectrum could then be recorded on the detector with the microscope set to diffraction mode. This microscope has two SAD planes (SAD1 and SAD2). In the present study, the upper (SAD1) plane was used.

The device was tested by using generation holograms in the condenser aperture plane to create electron beams with known OAM states [27]. The generation holograms were nanopatterned SiN membranes, in which thickness modulations were used to create intended phase distributions. With the image corrector unit turned off, an effective focal distance of $f \approx 530 \mathrm{~mm}$ could be achieved. A voltage of $V_{c}=-9.1 \mathrm{~V}$ applied to the main needle corresponded to a scale factor $s \approx 3 \mu \mathrm{rad}$. The lateral needles of element $S 1$ were kept at $V_{L}=-0.5 V_{c}$. For the $S 2$ element, the needles were biased with alternating voltages $\left|V_{S 2}\right|=16 \mathrm{~V}$ (with $8 \mathrm{~V}$ on the external ones). A change of applied bias could be compensated by a lateral shift of the beam. The alignment of the setup was found to be demanding, as the diffraction plane of element $S 1$ had to be matched in position, rotation, and magnification to element $S 2$. Under standard working conditions, a rotation angle of $\sim 23^{\circ}$ was measured between the objective aperture and the SAD1 aperture. This angle was compensated by designing a rotated element $S 2$, with the residual rotation adjusted by applying a minor excitation to the adaptor and transfer lenses of the aberration corrector. The magnification introduced by element $S 1$ was adjusted by tuning the bias of the main $S 1$ needle (with the voltages of the lateral needles tuned accordingly). The position of the diffraction pattern on element $S 2$ was adjusted by using the image shift coils.

Figures 2(b)-2(d) show the evolution of an electron beam from a generation hologram mounted in the condenser aperture plane (reproduced almost exactly at the entrance of the $S 1$ element) to the diffraction plane of element $S 1$. The images show unwrapping of the vortex into Cartesian coordinates and finally an OAM spectrum. In Fig. 2(c), the logarithmic radial coordinate is on the horizontal axis, while the azimuthal coordinate is on the vertical axis. If element $S 2$ works perfectly, then the result is expected to comprise vertically separated peaks that describe the OAM spectrum of the beam. Slight bending is observed in practice, perhaps as a result of residual charging of the needle or imperfect centering of the needle on the optical axis.

Experimental spectra corresponding to different electron beam combinations are shown in Fig. 3 for synthetic
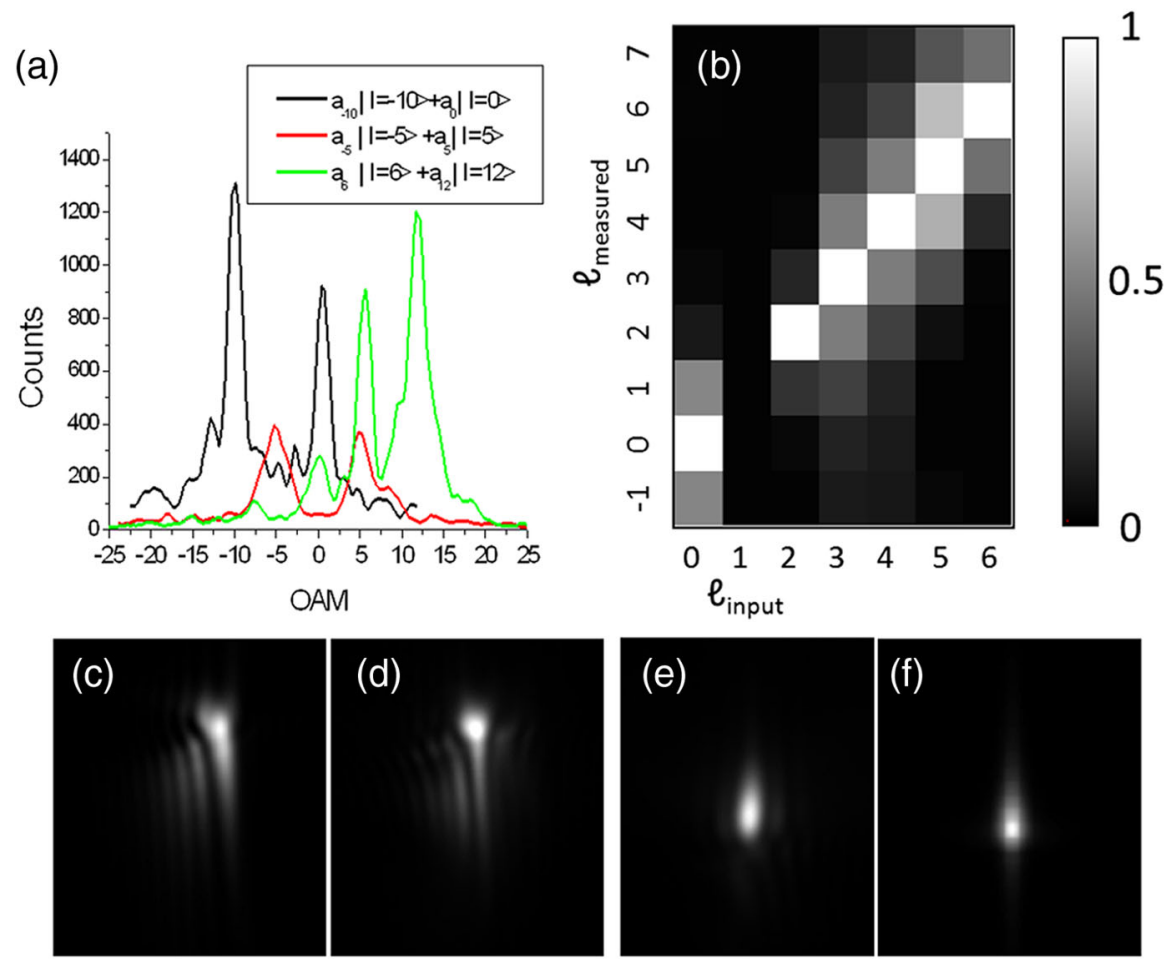

FIG. 3. (a) Experimentally measured OAM spectra for three test electron beams with the indicated nominal OAM compositions. (b) Experimental evaluation of cross talk between the nominal OAM value and the measured distribution. The figure is normalized so that the maximum value in each column is 1 . No data are available for $\ell_{\text {input }}=1$ since no hologram with $\ell_{\text {input }}=1$ was fabricated (it would have produced a confused spectrum with no possibility of internal calibration). (c),(e) Experimental and (d),(f) simulated OAM spectra for a "standard" $(\ell=0)$ beam. A match is obtained for $(\mathrm{c}),(\mathrm{d})$ incomplete and (e),(f) near complete compensation between diffraction from $S 1$ and the phase of $S 2$. (See text for details). 
holograms with the indicated nominal OAM decompositions. A near-quantitative comparison with an independent evaluation (based on image analysis, as in Ref. [54]) of the OAM composition for a superposition of beams with $l= \pm 5$ is reported in the Supplemental Material, alongside secondary electron microscopy (SEM) images of generation holograms [53]. The experimental cross talk between different OAM channels is shown in Fig. 3(b) for input beams with values of $\ell$ between 0 and 6 . The values of cross talk depend on the specific details of each experiment.

The OAM resolution $\Delta \ell$, which is defined here as the full width at half maximum of the spectrum, is found to be between 1.5 and 2, compared to an optimal value of unity [30]. This discrepancy is thought to result from imperfect alignment, while the background under each peak is likely to result from slight mismatch between the size of the diffraction pattern of element $S 1$ and the size of element $S 2$. The experimental OAM spectrum corresponding to a "standard" (azimuthally uniform) beam shown in Fig. 3(c) is reproduced nearly perfectly in the simulation shown in Fig. 3(d) by assuming a $1.1 \%$ error in the excitation of $S 1$ and an $80 \mathrm{~nm}$ lateral misalignment between the two sorting elements. (See the Supplemental Material [53]). The figure also shows a comparison between experiment and simulation for the case of near complete correction. A more thorough comparison between simulations and experiments will be presented in a separate paper [55].

A standard beam obtained using a conventional condenser aperture should have an azimuthally uniform wave front. The beam that enters the sorter is almost exactly in a plane conjugate to that of the condenser aperture and should decompose to only an $\ell=0$ component. The observed fringes result from misalignment. The absence of practically any material in the electron beam path (excluding the sorter 1 electrode) should rule out a spurious effect from the generation hologram. The simulations shown in Fig. 3(d) and an analytical model presented in the Supplemental Material [53] suggest that this mismatch is also responsible for the slight loss of resolution in the peaks. On the assumption that the phase mismatch is the primary limiting factor, two solutions can be proposed to improve the resolution.

(1) Since the phase mismatch is proportional to the phase gradient, the OAM resolution can be improved by reducing $s$, i.e., the voltage applied to element $S 1$, while maintaining the stationary phase condition $(s R / \lambda) \gg \ell_{\max }$ [31], where $R$ is the size of the beam at the entrance of the sorter and $\ell_{\max }$ is the maximum value of OAM to be measured. If this approach is used, then the product $s f$ must be matched to the periodicity of $S 2$, which is fixed by the geometry of the $S 2$ electrodes. For this reason, we increased the focal length by switching off the aberration corrector.

(2) In order to limit the phase gradient (and considering the geometric imperfection of sorter 1), it helps to limit the angle of acceptance of the sorter, for example, by using an aperture. In our tests without a sample, we simply reduced the convergence angle for this purpose.

Given the need for precise alignment, we believe that automatic diagnosis and alignment will be required regularly to reduce shift and magnification errors, in a manner similar to that used in adaptive optics in telescopes [56]. (See Ref. [57], and references therein).

In summary, we have described an OAM sorter for electron beams that is based on electrostatic phase elements and makes use of multiple-electrode-based control of electron wave functions. Experimental OAM spectra of test beams are found to be consistent with predictions. The OAM resolution is measured to be between $\Delta \ell \approx 1.5$ and 2 . In the future, improved alignment using computer-based diagnostics is expected to provide an optimal resolution of $\Delta \ell=1$ with a reduced background signal. The attainment of an ideal OAM resolution with the lossless efficiency of the electrostatic approach is expected to be important to achieve atomic-column-resolution magnetic measurements based on dichroism [58]. More generally, our demonstration of the use of MEMS-based technology to fabricate new components for charged particle optics offers a breakthrough for the measurement of previously inaccessible physical quantities, which are not limited to orbital angular momentum [52].

The authors acknowledge the support of the European Union's Horizon 2020 Research and Innovation Programme under Grant Agreement No. 766970 Q-SORT (H2020FETOPEN-1-2016-2017). This project has received funding from the European Union's Horizon 2020 Research and Innovation Programme (Grant No. 823717, project "ESTEEM3"). E. K. acknowledges support from an Ontario Early Researcher Award (ERA), and Canada Research Chairs (CRC) Program.

* Corresponding author. enzo.rotunno@cnr.it Corresponding author. vincenzo.grillo@cnr.it

[1] E. Ruska, Das entstehen des elektronenmikroskops und der elektronenmikroskopie (Nobel-Vortrag), Angew. Chem. 99, 611 (1987).

[2] R. Courtland, The microscope revolution that's sweeping through materials science, Nature (London) 563, 462 (2018).

[3] Y. Jiang, Z. Chen, Y. Han, P. Deb, H. Gao, S. Xie, P. Purohit, M. W. Tate, J. Park, S. M. Gruner, V. Elser, and D. A. Muller, Electron ptychography of 2D materials to deep subÅngström resolution, Nature (London) 559, 343 (2018).

[4] L. Jin, J. Barthel, C.-L. Jia, and K. W. Urban, Atomic resolution imaging of $\mathrm{YAlO}_{3}$ : Ce in the chromatic and spherical aberration corrected PICO electron microscope, Ultramicroscopy 176, 99 (2017).

[5] E. Yücelen, I. Lazić, and E. G. T. Bosch, Phase contrast scanning transmission electron microscopy imaging of light 
and heavy atoms at the limit of contrast and resolution, Sci. Rep. 8, 2676 (2018).

[6] S. de Graaf, J. Momand, C. Mitterbauer, S. Lazar, and B. J. Kooi, Resolving hydrogen atoms at metal-metal hydride interfaces, Sci. Adv. 6, eaay4312 (2020).

[7] P. A. Midgley and R. E. Dunin-Borkowski, Electron tomography and holography in materials science, Nat. Mater. 8, 271 (2009).

[8] S. Lopatin, B. Cheng, W.-T. Liu, M.-L. Tsai, J.-H. He, and A. Chuvilin, Optimization of monochromated TEM for ultimate resolution imaging and ultrahigh resolution electron energy loss spectroscopy, Ultramicroscopy 184, 109 (2018).

[9] D. Rossouw and G. A. Botton, Plasmonic Response of Bent Silver Nanowires for Nanophotonic Subwavelength Waveguiding, Phys. Rev. Lett. 110, 066801 (2013).

[10] R. Henderson, The potential and limitations of neutrons, electrons and x-rays for atomic resolution microscopy of unstained biological molecules, Q. Rev. Biophys. 28, 171 (1995).

[11] R. Henderson, Avoiding the pitfalls of single particle cryoelectron microscopy: Einstein from noise, Proc. Natl. Acad. Sci. U.S.A. 110, 18037 (2013).

[12] M. Haider, S. Uhlemann, E. Schwan, H. Rose, B. Kabius, and K. Urban, Electron microscopy image enhanced, Nature (London) 392, 768 (1998).

[13] O. L. Krivanek, N. Dellby, and A. R. Lupini, Towards sub- $\AA$ electron beams, Ultramicroscopy 78, 1 (1999).

[14] H. H. Rose, Historical aspects of aberration correction, J. Electron Microsc. 58, 77 (2009).

[15] K. Y. Bliokh, Y. P. Bliokh, S. Savel'ev, and F. Nori, Semiclassical Dynamics of Electron Wave Packet States with Phase Vortices, Phys. Rev. Lett. 99, 190404 (2007).

[16] M. Uchida and A. Tonomura, Generation of electron beams carrying orbital angular momentum, Nature (London) 464, 737 (2010).

[17] J. Verbeeck, H. Tian, and P. Schattschneider, Production and application of electron vortex beams, Nature (London) 467, 301 (2010).

[18] B. J. McMorran, A. Agrawal, I. M. Anderson, A. A. Herzing, H. J. Lezec, J. J. McClelland, and J. Unguris, Electron vortex beams with high quanta of orbital angular momentum, Science 331, 192 (2011).

[19] J. Harris, V. Grillo, E. Mafakheri, G. C. Gazzadi, S. Frabboni, R. W. Boyd, and E. Karimi, Structured quantum waves, Nat. Phys. 11, 629 (2015).

[20] B. J. McMorran, A. Agrawal, P. A. Ercius, V. Grillo, A. A. Herzing, T. R. Harvey, M. Linck, and J. S. Pierce, Origins and demonstrations of electrons with orbital angular momentum, Phil. Trans. R. Soc. A 375, 20150434 (2017).

[21] K. Y. Bliokh, I. P. Ivanov, G. Guzzinati, L. Clark, R. Van Boxem, A. Béché, R. Juchtmans, M. A. Alonso, P. Schattschneider, F. Nori, and J. Verbeeck, Theory and applications of free-electron vortex states, Phys. Rep. 690, 1 (2017).

[22] S. M. Lloyd, M. Babiker, G. Thirunavukkarasu, and J. Yuan, Electron vortices: Beams with orbital angular momentum, Rev. Mod. Phys. 89, 035004 (2017).

[23] D. Pohl, S. Schneider, P. Zeiger, J. Rusz, P. Tiemeijer, S. Lazar, K. Nielsch, and B. Rellinghaus, Atom size electron vortex beams with selectable orbital angular momentum, Sci. Rep. 7, 934 (2017).

[24] H. Larocque, I. Kaminer, V. Grillo, G. Leuchs, M. J. Padgett, R. W. Boyd, M. Segev, and E. Karimi, 'Twisted' electrons, Contemp. Phys. 59, 126 (2018).

[25] V. Grillo, E. Karimi, G. C. Gazzadi, S. Frabboni, M. R. Dennis, and R. W. Boyd, Generation of Nondiffracting Electron Bessel Beams, Phys. Rev. X 4, 011013 (2014).

[26] N. Voloch-Bloch, Y. Lereah, Y. Lilach, A. Gover, and A. Arie, Generation of electron Airy beams, Nature (London) 494, 331 (2013).

[27] V. Grillo, A. H. Tavabi, F. Venturi, H. Larocque, R. Balboni, G. C. Gazzadi, S. Frabboni, P.-H. Lu, E. Mafakheri, F. Bouchard, R. E. Dunin-Borkowski, R. W. Boyd, M. P. J. Lavery, M. J. Padgett, and E. Karimi, Measuring the orbital angular momentum spectrum of an electron beam, Nat. Commun. 8, 15536 (2017).

[28] V. Grillo, T. R. Harvey, F. Venturi, J. S. Pierce, R. Balboni, F. Bouchard, G. Carlo Gazzadi, S. Frabboni, A. H. Tavabi, Z.-A. Li, R. E. Dunin-Borkowski, R. W. Boyd, B. J. McMorran, and E. Karimi, Observation of nanoscale magnetic fields using twisted electron beams, Nat. Commun. 8, 689 (2017).

[29] G. Guzzinati, A. Béché, D. McGrouther, and J. Verbeeck, Prospects for out-of-plane magnetic field measurements through interference of electron vortex modes in the TEM, J. Opt. 21, 124002 (2019).

[30] B. J. McMorran, T. R. Harvey, and M. P. J. Lavery, Efficient sorting of free electron orbital angular momentum, New J. Phys. 19, 023053 (2017).

[31] G. Pozzi, V. Grillo, P.-H. Lu, A. H. Tavabi, E. Karimi, and R. E. Dunin-Borkowski, Design of electrostatic phase elements for sorting the orbital angular momentum of electrons, Ultramicroscopy 208, 112861 (2020).

[32] A. H. Tavabi, M. Beleggia, V. Migunov, A. Savenko, O. Öktem, R. E. Dunin-Borkowski, and G. Pozzi, Tunable ampere phase plate for low dose imaging of biomolecular complexes, Sci. Rep. 8, 5592 (2018).

[33] M. Zanfrognini, E. Rotunno, S. Frabboni, A. Sit, E. Karimi, U. Hohenester, and V. Grillo, Orbital angular momentum and energy loss characterization of plasmonic excitations in metallic nanostructures in TEM, ACS Photonics 6, 620 (2019).

[34] E. Rotunno, M. Zanfrognini, S. Frabboni, J. Rusz, R. E. Dunin Borkowski, E. Karimi, and V. Grillo, Orbital angular momentum resolved electron magnetic chiral dichroism, Phys. Rev. B 100, 224409 (2019).

[35] M. Zanfrognini, E. Rotunno, J. Rusz, R. E. D. Borkowski, E. Karimi, S. Frabboni, and V. Grillo, Sum rules in zone axis STEM-orbital angular momentum resolved electron magnetic chiral dichroism, Phys. Rev. B 102, 184420 (2020).

[36] F. S. Hage, R. J. Nicholls, J. R. Yates, D. G. McCulloch, T. C. Lovejoy, N. Dellby, O. L. Krivanek, K. Refson, and Q. M. Ramasse, Nanoscale momentum-resolved vibrational spectroscopy, Sci. Adv. 4, eaar7495 (2018).

[37] R. Senga, K. Suenaga, P. Barone, S. Morishita, F. Mauri, and T. Pichler, Position and momentum mapping of vibrations in graphene nanostructures, Nature (London) 573, 247 (2019). 
[38] C. A. Guido, E. Rotunno, M. Zanfrognini, S. Corni, and V. Grillo, Exploring the azimuthal symmetries of electronic transitions in molecular and biomolecular systems by swift electrons, arXiv:2005.07440.

[39] G. Bertoni, E. Rotunno, A. Tavabi, M. Zanfrognini, P. Rosi, S. Frabboni, E. Karimi, R. Dunin-Borkowski, and V. Grillo, Combination of electron energy-loss spectroscopy and orbital angular momentum spectroscopy. Applications to electron magnetic chiral dichroism, plasmon-loss, and coreloss, Microsc. Microanal. 26, 1752 (2020).

[40] K. X. Nguyen, Y. Jiang, M. C. Cao, P. Purohit, A. K. Yadav, P. García-Fernández, M. W. Tate, C. S. Chang, P. AguadoPuente, J. Íñiguez, F. Gomez-Ortiz, S. M. Gruner, J. Junquera, L. W. Martin, R. Ramesh, and D. A. Muller, Transferring orbital angular momentum to an electron beam reveals toroidal and chiral order, arXiv:2012.04134.

[41] F. Troiani, E. Rotunno, S. Frabboni, R. B. G. Ravelli, P. J. Peters, E. Karimi, and V. Grillo, Efficient molecule discrimination in electron microscopy through an optimized orbital angular momentum sorter, Phys. Rev. A 102, 043510 (2020).

[42] K. Saitoh, Y. Hasegawa, K. Hirakawa, N. Tanaka, and M. Uchida, Measuring the Orbital Angular Momentum of Electron Vortex Beams Using a Forked Grating, Phys. Rev. Lett. 111, 074801 (2013).

[43] G. Guzzinati, L. Clark, A. Béché, and J. Verbeeck, Measuring the orbital angular momentum of electron beams, Phys. Rev. A 89, 025803 (2014).

[44] L. Clark, A. Béché, G. Guzzinati, and J. Verbeeck, Quantitative measurement of orbital angular momentum in electron microscopy, Phys. Rev. A 89, 053818 (2014).

[45] R. Shiloh, Y. Tsur, R. Remez, Y. Lereah, B. A. Malomed, V. Shvedov, C. Hnatovsky, W. Krolikowski, and A. Arie, Unveiling the Orbital Angular Momentum and Acceleration of Electron Beams, Phys. Rev. Lett. 114, 096102 (2015).

[46] H. Larocque, F. Bouchard, V. Grillo, A. Sit, S. Frabboni, R. E. Dunin-Borkowski, M. J. Padgett, R. W. Boyd, and E. Karimi, Nondestructive Measurement of Orbital Angular Momentum for an Electron Beam, Phys. Rev. Lett. 117, 154801 (2016).

[47] T. R. Harvey, V. Grillo, and B. J. McMorran, Stern-Gerlachlike approach to electron orbital angular momentum measurement, Phys. Rev. A 95, 021801(R) (2017).
[48] Y. Noguchi, S. Nakayama, T. Ishida, K. Saitoh, and M. Uchida, Efficient Measurement of the Orbital-AngularMomentum Spectrum of an Electron Beam via a Dammann Vortex Grating, Phys. Rev. Applied 12, 064062 (2019).

[49] A. Lubk, G. Guzzinati, F. Börrnert, and J. Verbeeck, Transport of Intensity Phase Retrieval of Arbitrary Wave Fields Including Vortices, Phys. Rev. Lett. 111, 173902 (2013).

[50] T. Schachinger, S. Löffler, A. Steiger-Thirsfeld, M. StögerPollach, S. Schneider, D. Pohl, B. Rellinghaus, and P. Schattschneider, EMCD with an electron vortex filter: Limitations and possibilities, Ultramicroscopy 179, 15 (2017).

[51] G. C. G. Berkhout, M. P. J. Lavery, J. Courtial, M. W. Beijersbergen, and M. J. Padgett, Efficient Sorting of Orbital Angular Momentum States of Light, Phys. Rev. Lett. 105, 153601 (2010).

[52] G. Ruffato, E. Rotunno, and V. Grillo, A general framework for conformal transformations in electron optics, arXiv: 2003.09635.

[53] See Supplemental Material at http://link.aps.org/supplemental/ 10.1103/PhysRevLett.126.094802 for details about formulas, holograms, simulations, for an analytical model of the "mismatch effect" and an independent check of the validity of the OAM decomposition.

[54] V. Grillo, G. C. Gazzadi, E. Mafakheri, S. Frabboni, E. Karimi, and R. W. Boyd, Holographic Generation of Highly Twisted Electron Beams, Phys. Rev. Lett. 114, 034801 (2015).

[55] E. Rotunno, A. H. Tavabi, P. Rosi, S. Frabboni, P. Tiemeijer, R. E. Dunin-Borkowski, and V. Grillo, Alignment of electron optical beam shaping elements using a convolutional neural network, arXiv:2101.04358.

[56] J. Y. Wang, Optical resolution through a turbulent medium with adaptive phase compensations, J. Opt. Soc. Am. 67, 383 (1977).

[57] J. Verbeeck, A. Béché, K. Müller-Caspary, G. Guzzinati, M. A. Luong, and M. Den, Hertog, demonstration of a $2 \times 2$ programmable phase plate for electrons, Ultramicroscopy 190, 58 (2018).https://doi.org/10.1038/s41578-020-00275-8

[58] J. C. Idrobo, A new resolution quest in electron microscopy, Nat. Rev. Mater. 6, 100 (2021). 